\title{
Evaluación de la potencia de un molino de martillos al procesar bagazo de caña de azúcar
}

\author{
A hammer mill power evaluation from sugarcane bagasse processing
}

\section{Sneider Alejandro Gil P. ; Albeiro Espinosa B. ${ }^{2}$}

1. Ingeniero Mecánico, Universidad Nacional de Colombia. Medellín, Colombia, sagilpa@unal.edu.co.

2. Ph.D. Profesor Asociado, Universidad Nacional de Colombia. Medellín, Colombia, aespinos@unal.edu.co.

Citar: Gil, S. \& Espinosa, A. (2019). Evaluación de la potencia de un molino de martillos al procesar bagazo de caña de azúcar. Revista de Ciencias Agrícolas. 36(1): 93-108. doi: http://dx.doi.org/10.22267/ rcia.193601.101

Recibido: mayo 22 de $2018 . \quad$ Aceptado: febrero 14 de 2019.

\section{RESUMEN}

La producción de panela es un renglón importante de la economía colombiana, esta agroindustria es bajamente tecnificada y usa máquinas mecánicamente ineficientes que ocasionan altos consumos de energía. Este trabajo pretende evaluar el consumo de potencia de un molino de martillos durante el procesamiento de bagazo de caña de azúcar. Se empleó una pinza voltiamperimétrica que graba la información de corriente, voltaje y ángulo de fase durante un periodo de tiempo, simultáneamente se agregan cargas controladas de bagazo a intervalos regulares. Se encontró que existe una relación entre la carga y el consumo de potencia, la cual se ajustó mediante un modelo cuadrático cuyo coeficiente de correlación es 0,99 .

Palabras clave: Molino, desechos agroindustriales, potencia, modelo de carga, relación potenciabagazo, máquinas agrícolas, eficiencia en máquinas. 


\begin{abstract}
The Panela production is an important item of the Colombian economy. This agricultural industry has a low technification and uses mechanically inefficient machines that cause high- energy consumption. This work aims to check the power consumption of a hammer mill during the sugarcane bagasse processing. A Voltiamperimetric clamp recorded the current, voltage and phase angle information for a period, simultaneously adding controlled loads of bagasse at regular intervals. It was found that there is a relationship between the load and the power consumption, which was adjusted by a quadratic model whose correlation coefficient is 0.99 .
\end{abstract}

Keywords: Mill, agricultural industry waste, power, loading model, bagasse-power relation, agricultural machines, machines efficiency.

\section{INTRODUCCIÓN}

En Colombia, la industria panelera tiene gran impacto económico, ya que, de ella se benefician muchas familias campesinas al ser un producto tradicional y de elaboración artesanal. Después del café, la panela ocupa el segundo lugar en la industria agrícola de Colombia, y también ocupa el segundo puesto a nivel internacional, siendo la India el primero en producción ( Leiva al., 2006; Murcia-Pardo y Ramírez-Durán, 2017).

La industria panelera, al igual que el resto de la industria agrícola en el mundo, posee baja tecnificación. La causa es el predominio del empirismo en la agricultura debido a fenómenos culturales y el conformismo de su funcionalidad (Guerrero \& Escobar, 2015 ; Cañizares, 2015). En consecuencia, se ha generado un crecimiento débil en las fuerzas productivas que ha generado bajo crecimiento económico (Pérez y Cortés, 2017); debido a ello, se ha recomendado aumentar la tecnificación en el campo, lo cual mejoraría la eficiencia en la producción agrícola y reduciría costos (Guerrero \& Escobar, 2015; Santillan-Fernandez et al., 2014). Hoy en día, sólo el 2\% de la industria panelera de Colombia poseen alta tecnificación, es decir, 460 de los 23000 trapiches. En cambio, 3450 poseen algo de tecnificación y son considerados de mediana producción, el resto, con cerca del 83\% de los productores de panela (19090 trapiches), son considerados como productores menores debido a que poseen poca o ninguna tecnificación (Leiva y Arbeláez, 2006; Flórez, 2013; Durán et al, 2014).

Usar el bagazo de caña como combustible en forma de pellets permitiría reducir el impacto ambiental generado por el uso de combustibles fósiles (Rodríguez-Pérez et al., 2014). Además, la cantidad de energía por unidad de volumen en el bagazo en forma de pellets es mayor que en su estado a la salida del molino (Gracia et al, 2016; Vega-Nieva et al., 2015). La baja tecnificación genera consecuencias negativas en el ambiente, al usar combustibles contaminantes o combustibles con bajo poder calorífico como la leña. En este último caso, el daño es mayor por deforestación en los bosques (García, 2004; Vera, 2014). Los pellets son una buena alternativa que permite dar uso al bagazo y así reducir el impacto ambiental, esto se debe a que el pellet posee mayor poder calorífico que el bagazo sin procesar, ocupa menos espacio y evita problemas ambientales (Tiwari, 2011). Además, de esta forma, su combustión arroja pocos contaminantes a la atmósfera (Ballester, 2005; Fredes, 2014).

Convertir el bagazo de caña en pellets requiere de un proceso de molienda que como se 
expresó previamente es ineficiente. Por sus características, el molino de martillos es la herramienta más apropiada para procesar el bagazo de caña de azúcar, la cual, es bastante fibrosa. El uso del molino de martillos genera problemas adicionales como: ruido, polución y baja eficiencia de molido(Elias et al, 2014; Bradshaw, 2009; Arpi et al, 2010). Ésta máquina posee una eficiencia de molido máximo de 28,96\% (Bitra et al., 2009), aunque de manera experimental se llegó a una eficiencia de corte de $66,71 \%$, siendo aún bastante ineficiente (Jannasch et al., 2001). Existen múltiples variables que pueden afectar la eficiencia del molino, una de ellas es la geometría del martillo (Yancey et al., 2013).

A la fecha, no ha habido estudios sobre la caracterización del consumo de potencia de un molino de martillos en operación, que permita construir un modelo del consumo de potencia. Un modelo matemático para un triturador de corte vertical es usado con rastrojo (Pérez y Garbati, 2008), donde la variable principal del modelo de consumo de potencia es la velocidad angular del rotor del órgano de corte. Un modelo para un molino forrajero de tambor se propone en Martínez et al. (2004) y Valdés et al. (2012). En este, se consideran las variables de ángulo de ataque y las fuerzas de corte. Se han hecho algunos estudios de modelos matemáticos para el consumo de potencia de entrada en otras máquinas agrícolas diferentes a molinos, principalmente tractores. Un primer estudio desarrolla un modelo donde se tiene en cuenta variables como el peso y la vida económica del mismo (Ozkan et al., 2004). Un modelo conocido como el método Nebraska, es usado para conocer el consumo de potencia de los tractores, teniendo en cuenta factores como velocidades de operación, presión y caudal del aceite al interior de la bomba, entre otras variables (Romantchik-Kriuchkov et al, 2015; Vargas et al., 2013; Ayala et al., 2013). Para el caso de las turbinas eólicas, el modelo matemático usa ecuaciones simples de movimiento. Algunas variables son la densidad del aire, su velocidad, el área de los bordes de los álabes, entre otros (Chen y Spooner, 2001).

El objetivo de este artículo es presentar un modelo de potencia en función de la carga de bagazo a procesar, de esta forma se busca evaluar la potencia consumida por un molino de martillos usado para triturar el bagazo de caña de azúcar.

\section{MATERIALES Y MÉTODOS}

La potencia en el molino fue cuantificada midiendo el voltaje y el amperaje en el motor empleando una pinza voltiamperimetrica que graba en el tiempo las variables de voltaje, corriente y ángulo de fase. Se realizaron experimentos por lotes con entradas al molino de una determinada cantidad de bagazo cada 60 segundos. La cantidad de bagazo fue medida utilizando una pesa gramera EK 5015 Electronic Kitchen Scale (Hopex Werkzeuge, Germany) con capacidad de $5 \mathrm{~kg}$ y con una resolución de 1g. La Tabla 1 muestra las características de la pinza voltiamperimétrica. La Tabla 2 describe las características del molino. En la Figura 1 se puede apreciar esta máquina. La Tabla 3 presenta las características generales del bagazo de caña. 
Tabla 1. Caracterización de la pinza voltiamperimétrica de marca UNI - T modelo UT 243

\begin{tabular}{ll}
\hline \multicolumn{1}{c}{ Descripción } & $150 \mathrm{~V}-600 \mathrm{~V}$ \\
\hline Rango de voltaje AC & $\pm(1,2 \%+5)$ \\
Precisión de voltaje AC & $0,1 \mathrm{~V}$ \\
Resolución de Voltaje AC & $5 \mathrm{M} \Omega$ \\
Impedancia de Entrada Voltaje AC & $40 \mathrm{~Hz}-80 \mathrm{~Hz}$ \\
Rango de Frecuencia & $\pm(0,5 \%+5)$ \\
Precisión de la frecuencia & $0,01 \mathrm{~Hz}$ \\
Resolución de la frecuencia & $50 \mathrm{~A}-1000 \mathrm{~V}$ \\
Rangos de Corriente AC & $\pm(2 \%+5)$ \\
Precisión de la corriente AC & $0,1 \mathrm{~A}($ para $50 \mathrm{~A}$ y $250 \mathrm{~A})-1 \mathrm{~A}$ (para $1000 \mathrm{~A})$ \\
Resolución de la corriente AC & $0^{\circ}-360^{\circ}$ \\
Localización del rango del ángulo de fase & $\pm 2^{\circ}$ \\
Ubicación del ángulo de fase de precisión & $1^{\circ}$ \\
Resolución de ángulo de fase de ubicación & $1 \sim 9999 \mathrm{KWh}$ \\
Rango de la potencia eléctrica & $\pm(3 \%+2)$ \\
Precisión de la energía eléctrica & $0,001 \mathrm{KWh}$ \\
Resolución de la energía eléctrica & $0,3 \sim 1$ \\
Rango del factor de potencia & $\pm 0,022$ \\
Precisión del factor de potencia & 0,001 \\
Resolución del factor de potencia &
\end{tabular}

Tabla 2. Caracterización del molino de martillos marca ASEA, modelo IEC 34 IP54 MT100L128-4

\begin{tabular}{lll}
\hline \multicolumn{1}{c}{ Descripción } & \multicolumn{1}{c}{ Valor } \\
\hline Clase & $\mathrm{F}$ \\
Potencia & $3,2 \mathrm{KW}$ \\
Velocidad angular & $1730 \mathrm{RPM}$ \\
VY: Voltaje en con conexión en estrella & 380 \\
Corriente $(\mathrm{VY})$ & $5,2 \mathrm{~A}$ \\
V $\Delta$ : Voltaje con conexión en delta & 220 \\
Corriente $(\mathrm{V} \Delta)$ & $9 \mathrm{~A}$ \\
Cos $\varphi$ & 0,76 \\
Dimensiones del martillos & $90 \mathrm{~mm} \times 38 \mathrm{~mm} \times 10 \mathrm{~mm}$ \\
Número de martillos & \multicolumn{1}{c}{15} \\
\hline
\end{tabular}




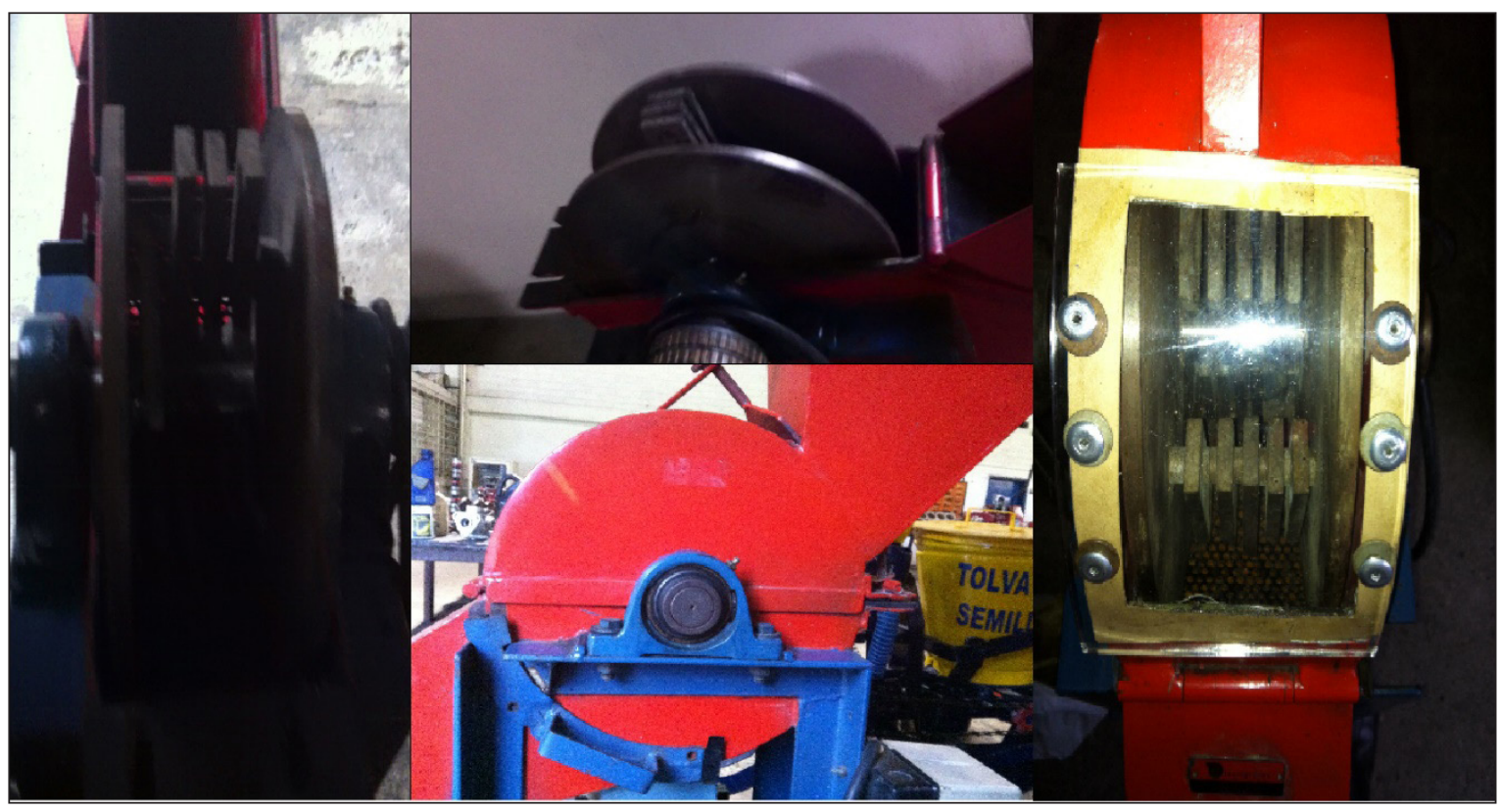

Figura 1. Molino de martillos marca ASEA, modelo IEC 34 IP54 MT100L128-4 visto de frontal y lateralmente con la carcasa abierta y cerrada.

Tabla 3. Características del bagazo de caña de azúcar

\begin{tabular}{ll}
\hline \multicolumn{1}{c}{ Propiedades físicas } & \multicolumn{1}{c}{ Valor promedio } \\
\hline Humedad a $105^{\circ} \mathrm{C}(\%)$ & $7,57 \pm 1,9 \times 10^{-1}$ \\
Densidad aparente $\left(\mathrm{kg} / \mathrm{m}^{3}\right)$ & $69,7 \pm 0,01$ \\
Densidad real $\left(\mathrm{kg} / \mathrm{m}^{3}\right)$ & $165,6 \pm 0,99$ \\
Porosidad & $0,60 \pm 1,4 \times 10^{-5}$ \\
Flotabilidad & Sí \\
\hline
\end{tabular}

Fuente: Martinez et al., 2014.

El montaje consiste en posicionar la pinza de tal manera que el cable unida a la fase con mayor corriente cruce en el medio, luego unir cada pinza de mango plástico a la fase que le corresponde, teniendo en cuenta que esto se hace en la caja donde está el sistema de encendido de la máquina, tal como se observa en la Figura 2. Lo descrito se hace para medir el voltaje, la corriente del motor y el ángulo de desfase, el equipo de medición envía toda la información con respecto al tiempo al computador, luego con la ecuación (Ec 1) se calcula la potencia de la máquina.

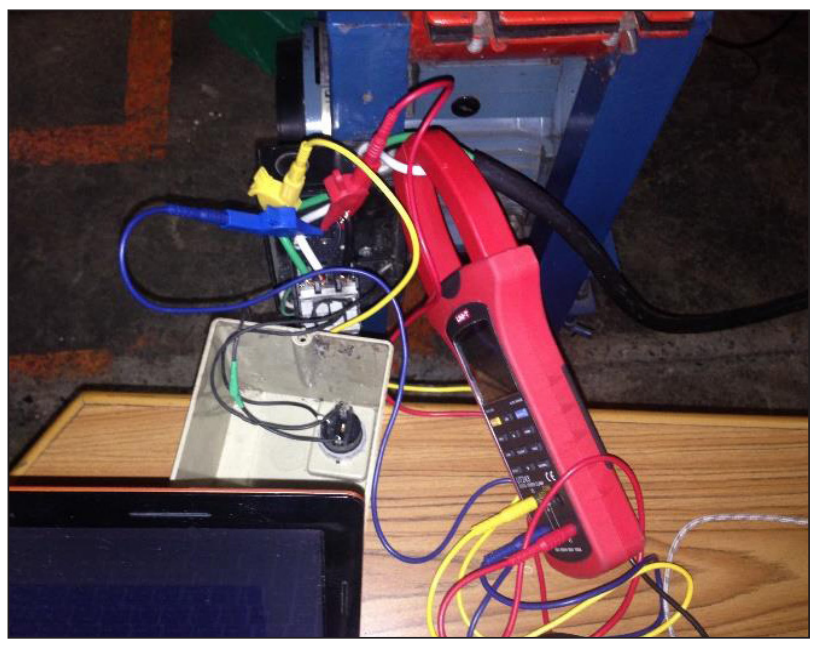

Figura 2. Montaje de la pinza sobre el cableado de transmisión de energía.

$$
\mathrm{P}=\mathrm{V} * \mathrm{I} * \operatorname{Cos}(\phi)
$$

Donde:

P: Potencia eléctrica del molino de martillos,

V: Voltaje del molino de martillos,

I: Corriente eléctrica de la misma máquina y $\operatorname{Cos}(\phi)$ (Coseno del ángulo de desfase entre el Voltaje y la corriente). 
Una vez realizado el montaje para medir la potencia y poner al molino funcionando en vacio, se le introduce una muestra de bagazo de caña de azúcar cada 60 segundos, tal como se describe en la Tabla 4, teniendo en cuenta que después de cada experimento la máquina se le hace limpieza para evitar que los residuos del experimento anterior generen sesgos.

Tabla 4. Descripción de cómo se arrojó las muestras de bagazo de caña de azúcar durante cada experimento.

\begin{tabular}{cccc}
\hline $\begin{array}{c}\text { Número de } \\
\text { experimento }\end{array}$ & $\begin{array}{c}\text { Cantidad de masa } \\
\text { a moler } \mathbf{( g )}\end{array}$ & $\begin{array}{c}\text { Número de } \\
\text { muestras }\end{array}$ & $\begin{array}{c}\text { Tiempo en el cual se } \\
\text { arroja la muestra }\end{array}$ \\
\hline 1 & 0 & 5 & Cada 60 segundos \\
2 & 12 & 5 & Cada 60 segundos \\
3 & 24 & 5 & Cada 60 segundos \\
4 & 48 & 5 & $\begin{array}{l}\text { Las tres primeras muestras cada } 60 \text { segundos, la } \\
\text { cuarta muestra a los } 120 \text { segundos y la última, 60 } \\
\text { segundos después }\end{array}$ \\
\hline
\end{tabular}

Finalmente, en cada experimento se pesó la cantidad de polvo fino que salió del molino de martillos a través de la criba. Esto permite conocer cuánto material fue procesado, cuanto material permaneció al interior del molino y cuál fue la eficiencia del procesamiento.

\section{RESULTADOS Y DISCUSIÓN}

Primer experimento. Sin tener en cuenta el momento de arranque, si se analiza la Figura $3 \mathrm{~A}$, se observa que el intervalo en que varía el coseno del ángulo de desfase con respecto al tiempo es pequeño, ya que el punto más bajo de la gráfica está en un poco más arriba de 0,96 y el pico más alto está en 0,98. En cuanto a la Figura 3C pasa algo similar, debido a que el voltaje se ubica entre el intervalo $125.5 \mathrm{~V}$ hasta $126 \mathrm{~V}$ durante el trascurso del tiempo. En cuanto a la Figura 3D, ésta no presenta cambios durante el tiempo. Si se analiza la Figura 3B, la cual se hizo usando la ecuación (Ec 1), se concluye que al tener unos intervalos tan pequeños tanto el Voltaje como el Coseno del ángulo de desfase, la potencia no presenta variaciones significativas con respecto al tiempo. 


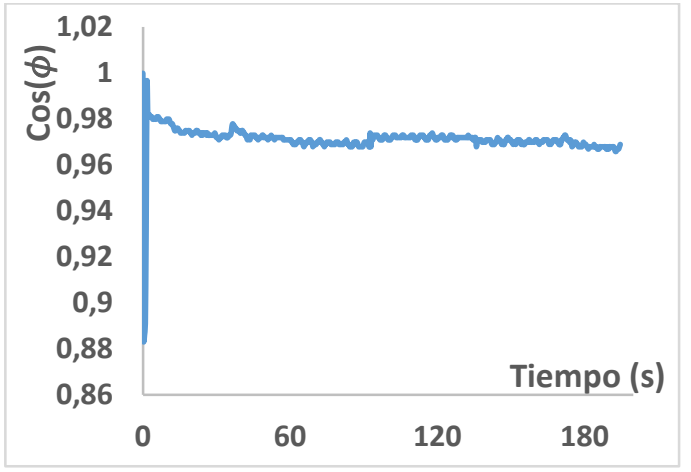

(A)

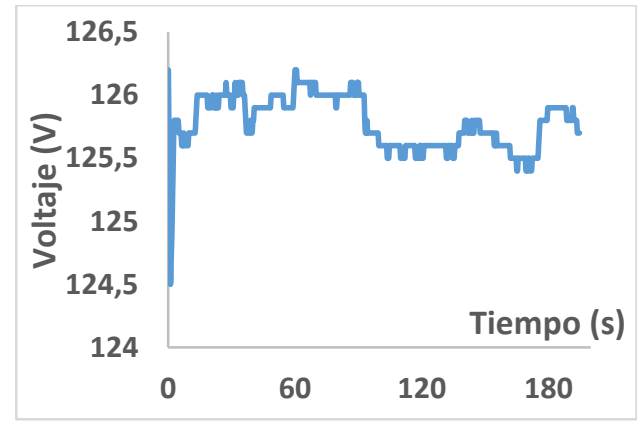

(C)

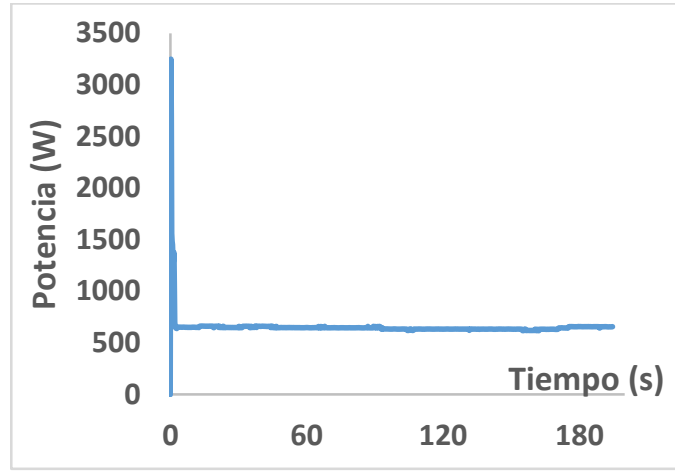

(B)

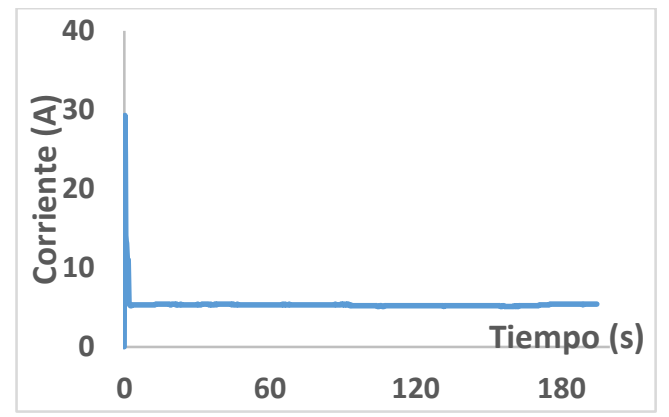

(D)

Figura 3. Resultados de cada variable medida con respecto al tiempo en el experimento 1.

Segundo experimento. Teniendo en cuenta que se desprecia el momento de arranque y que además están marcados con líneas rojas los instantes en que se introdujo las muestras de 12 g para ser trituradas, la Figura 4A se aprecia como decrece con respecto al tiempo, aunque con picos en los instantes posteriores al ingreso de las muestras en el molino. La Figura 4C se observa como el voltaje aumenta con el tiempo, en especial después de ingresar la cuarta muestra, donde el aumento es mayor. La Figura 4D no presenta cambios con respecto al tiempo. La Figura 4B, muestra los picos en los instantes después de que se introdujo las muestras al molino, esto se debe a los cambios en el Voltaje y el Coseno del ángulo de desfase; además se aprecia una estabilización de la potencia con el tiempo. Las variaciones del voltaje en son pequeños, razón por la cual influye poco en las magnitudes de la potencia; con la corriente ocurre lo mismo debido a que no presenta cambios en el tiempo, por ende, el que más influye con el comportamiento es el coseno del ángulo de desfase, razón por la cual las Figuras 4A y 4B son tan similares. 


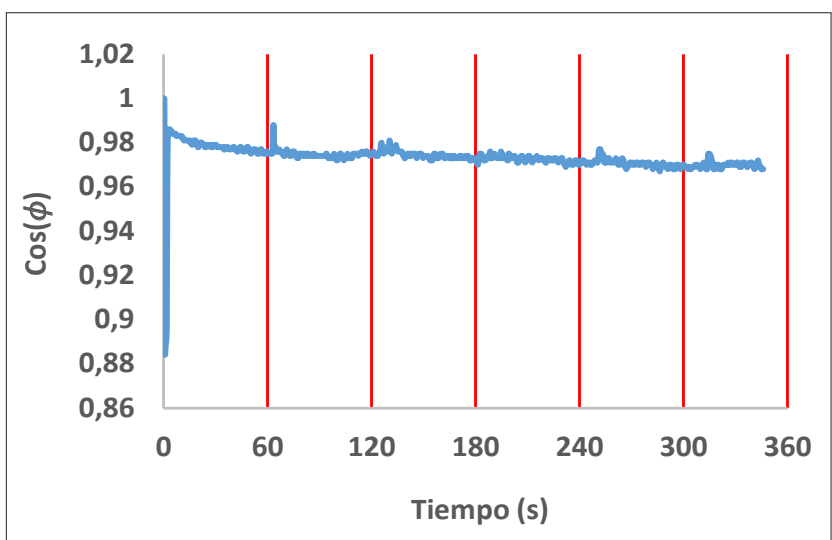

(A)

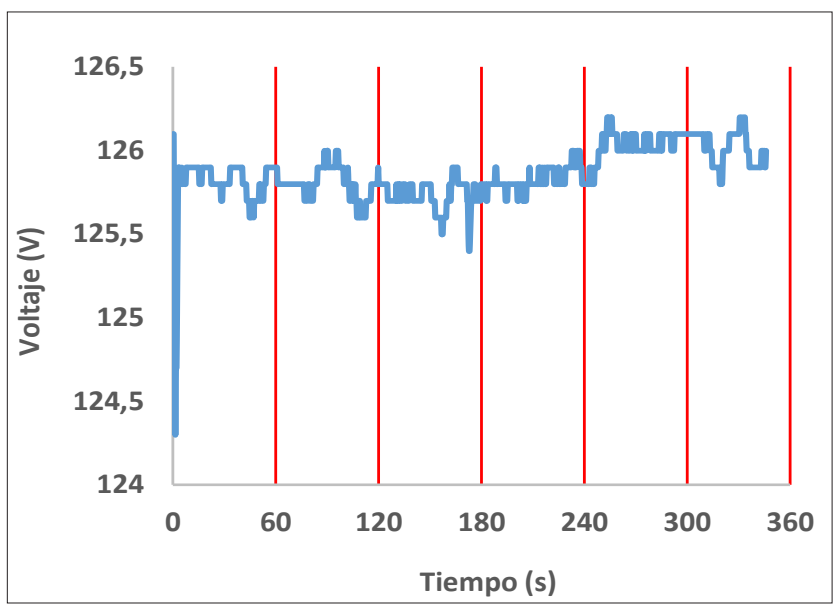

(C)

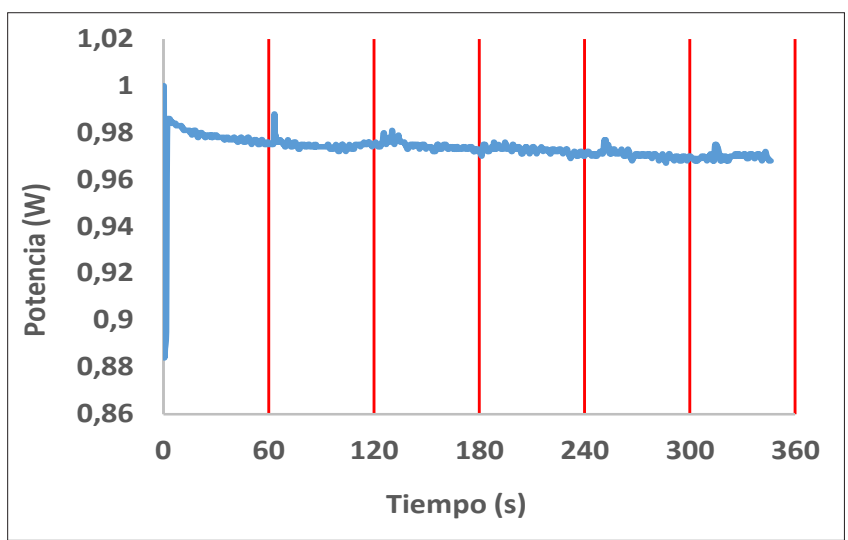

(B)

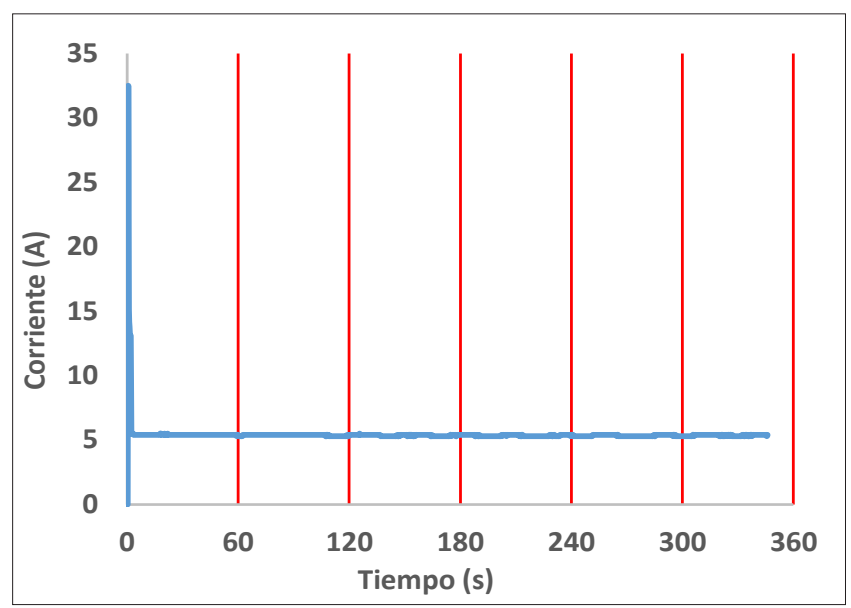

(D)

Figura 4. Resultados de las variables medidas en el segundo experimento con respecto al tiempo mientras se le adicionaba las muestras de $12 \mathrm{~g}$ a moler cada 60 segundos.

Tercer experimento. Para el siguiente análisis se debe tener en cuenta que el momento de arranque se desprecia y que además las líneas rojas denotan los instantes en los cuales las muestras de $24 \mathrm{~g}$ fueron arrojados en el molino para ser trituradas. La Figura 5A muestra como el coseno del ángulo de desfase va aumentando paulatinamente con respecto al tiempo, además de que hay picos que sobresalen en los instantes después de que las muestras son puestas en la máquina. La Figura 5C muestra como el voltaje se reduce con respecto al tiempo, en cambio la Figura 5D muestra que la corriente no presenta ningún cambio en el tiempo. La Figura 5B, muestra que el valor de la potencia se puede considerar estable con respecto al tiempo, ya que el valor del mismo no presentó variaciones significativas. 


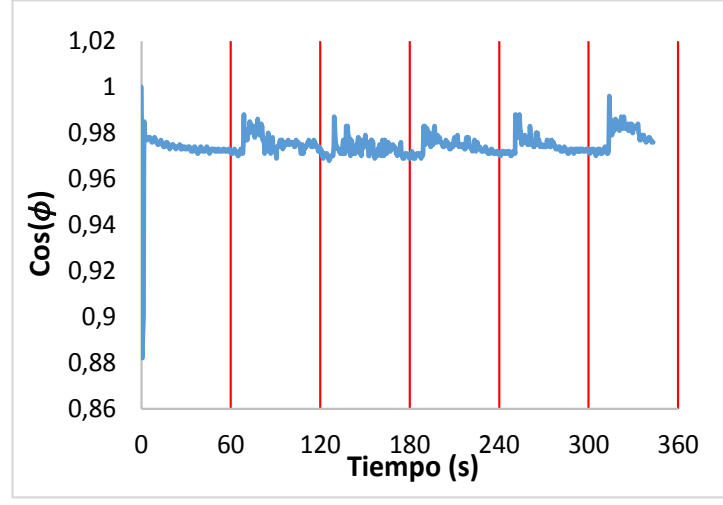

(A)

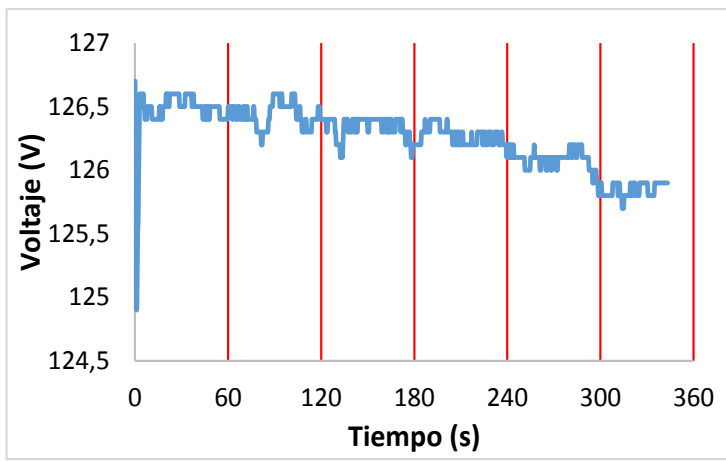

(C)

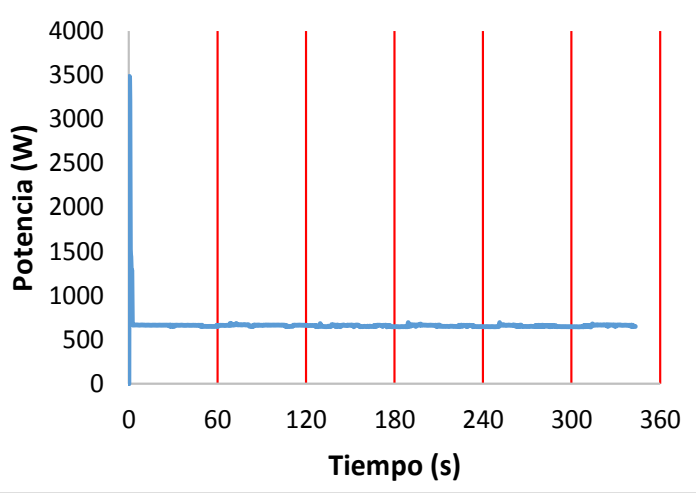

(B)

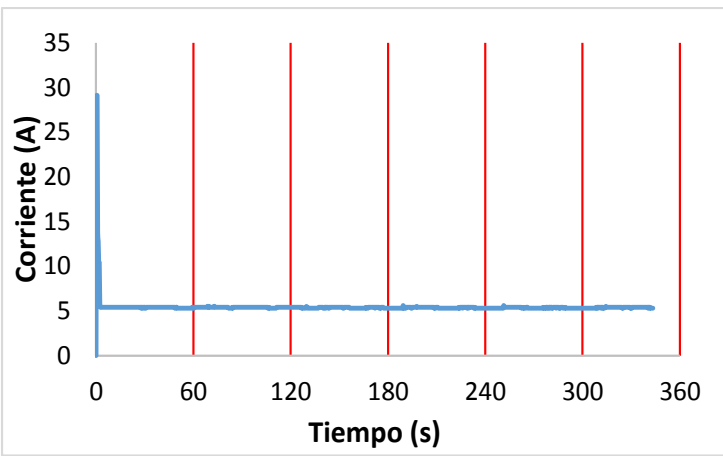

(D)

Figura 5. Resultados obtenidos en el tercer experimento a partir de las variables medidas con respecto al tiempo mientras se le adicionaba muestras de $24 \mathrm{~g}$ cada 60 segundos.

Cuarto experimento. Teniendo en cuenta que se despreció el momento de arranque durante el análisis y que se marcó con líneas rojas los instantes en los cuales las muestras de bagazo de $48 \mathrm{~g}$ fueron arrojados en el molino para ser triturados, la Figura 6A muestra como el Coseno del ángulo de desfase va aumentando mientras el molino iba triturando las muestras, y en el instante después de triturar la quinta muestra, este reduce hasta llegar al punto más bajo de la gráfica, en cambio en la Figura 6C se puede apreciar cómo va disminuyendo el voltaje con respecto al tiempo, aunque hay un pequeño aumento en el instante después de introducir la cuarta muestra, pero después de ese momento, sigue disminuyendo. En cuanto a la Figura 6D, por primera vez se observa cambios en la corriente, ocurriendo un aumento paulatino, además, se encontró picos en los instantes en que el molino empezó a triturar las muestras, pero en especial cuando se trituró la cuarta muestra, donde muestra un sobresalto, el cual continúa incluso cuando el molino tritura la quinta muestra. Los cambios en el voltaje, corriente y ángulos de desfase al introducir la cuarta muestra al molino, revelan que este tamaño de carga empieza a exigir mayor consumo de potencia y que la máquina comienza a saturarse. El motivo de todos estos cambios es por efecto de acumulación, debido a que, al ser unas muestras grandes para la capacidad de la máquina, ésta no trituraba en los 60 segundos toda la primera muestra, por ende, cuando se le introducía la siguiente, el molino trituraba la muestra recién arrojada más una parte de la muestra anterior. 


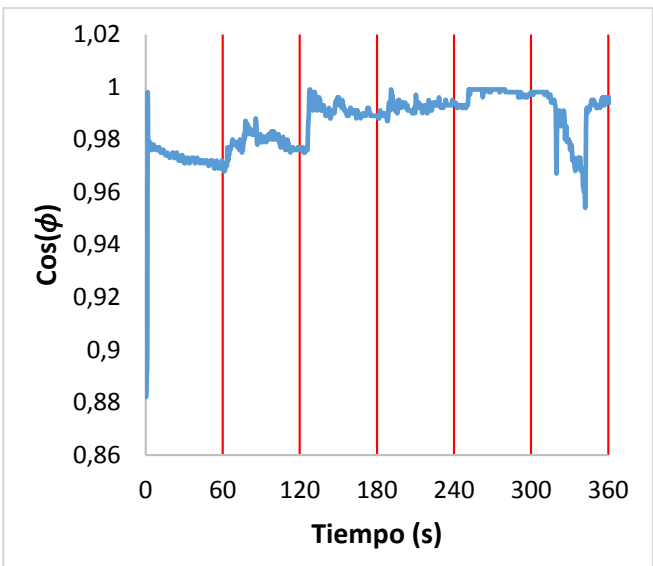

(A)

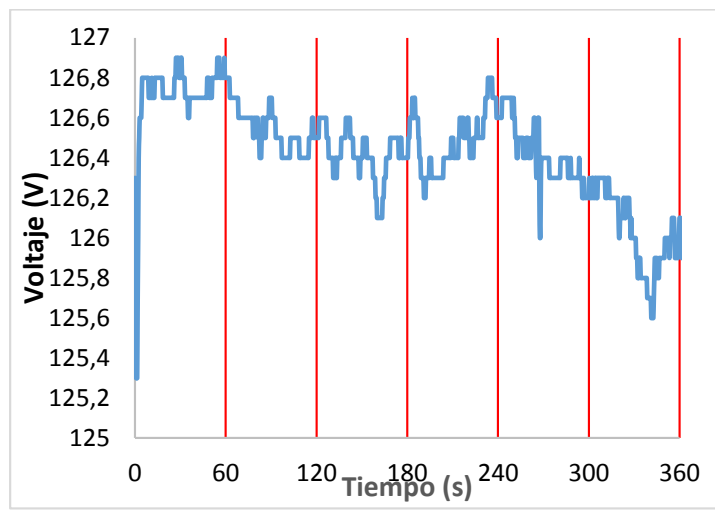

(C)

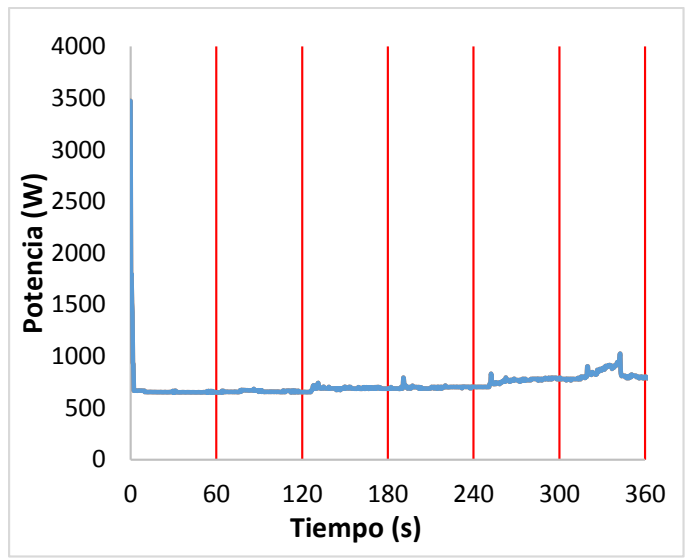

(B)

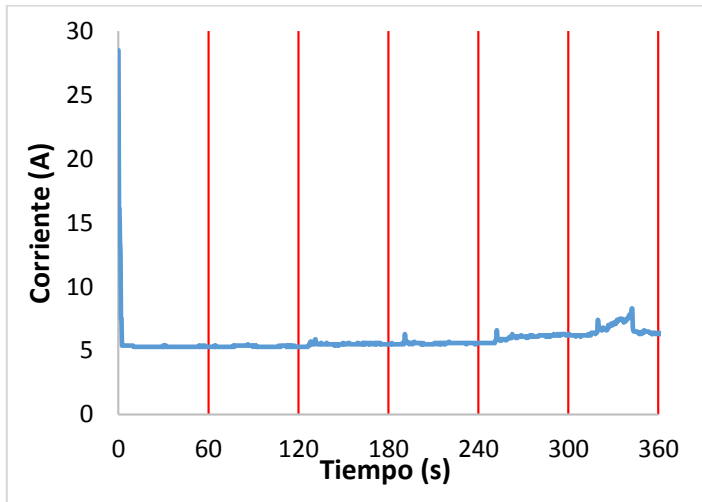

(D)

Figura 6. Resultado de los valores medidos en el cuarto experimento con respecto al tiempo e introduciendo en la molienda cinco muestras de $48 \mathrm{~g}$ cada 60 segundos.

Quinto experimento Hay que tener en cuenta que este es el experimento que tiene las muestras más pesadas con respecto a las anteriores, ya que cada una pesa $72 \mathrm{~g}$. Igual que en los casos anteriores, no se considera el momento de arranque y los momentos en que se ingresaron las muestras al molino están señaladas con líneas rojas en la Figura 7. En la Figura 7A se aprecia el incremento que tiene el coseno del ángulo de desfase después de triturar la primera muestra, En la Figura 7C se aprecia el incremento en el voltaje en el momento después de triturar la primera muestra, luego decrece paulatina con respecto al tiempo, obteniendo el valor más bajo de voltaje después de triturar la cuarta muestra, aunque hay un intento de incrementar después de triturar la quinta muestra, no llega alcanzar el punto más alto de la gráfica. En la Figura 7D se aprecia como la corriente, aunque trata de estar estable, hay pequeños picos después de triturar las primeras tres muestras, pero después de la cuarta muestra este incrementa considerablemente, incremento que continúa incluso después triturar la quinta muestra, alcanzando en esta el pico más alto. Basado en los resultados anteriores, si se analiza la Figura 7B, se aprecia como después de triturar las primeras tres muestras intenta estar estable, aunque haya picos en los instantes después de triturar las muestras descritas. Después de triturar la cuarta muestra y quinta muestra, se ve como la potencia aumenta considerablemente con respecto a todas las muestras anteriores, esto se debe al efecto de acumulación, ya que el voltaje y el ángulo del coseno de desfase van disminuyendo con el tiempo, pero en ese instante la corriente está aumentando de manera considerable. 


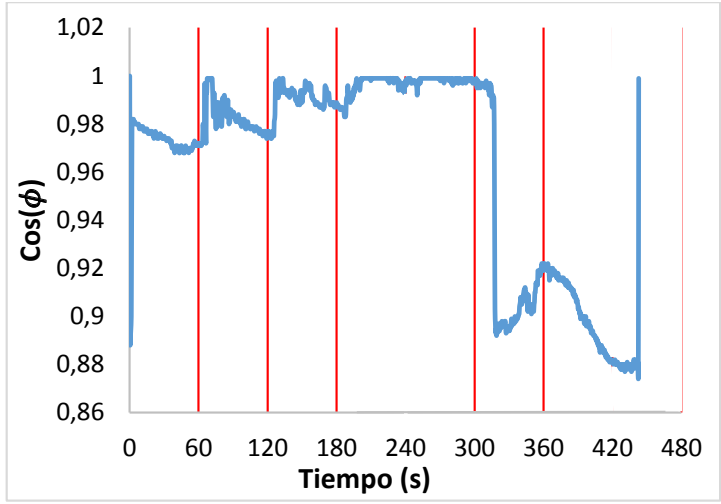

(A)

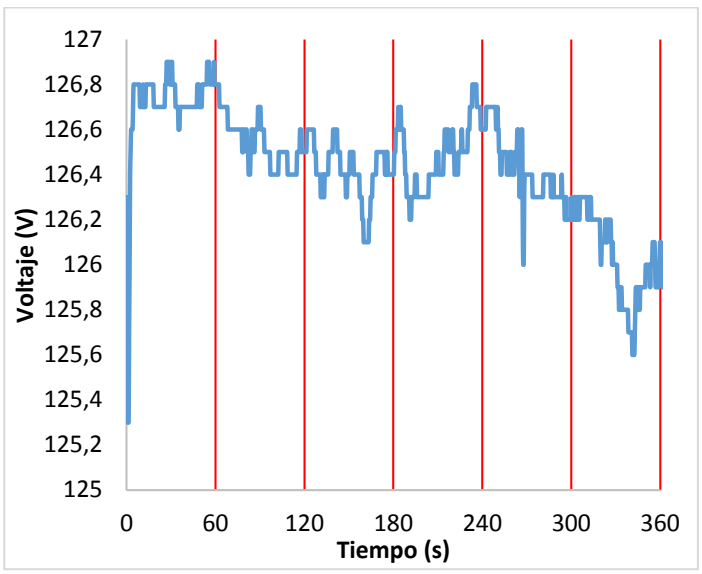

(C)

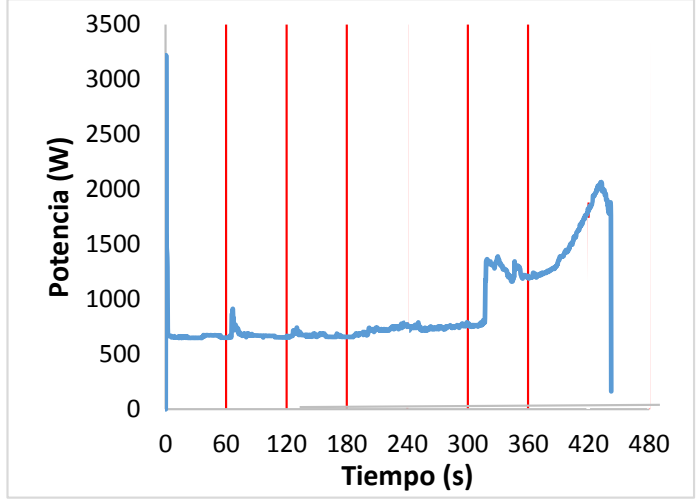

(B)

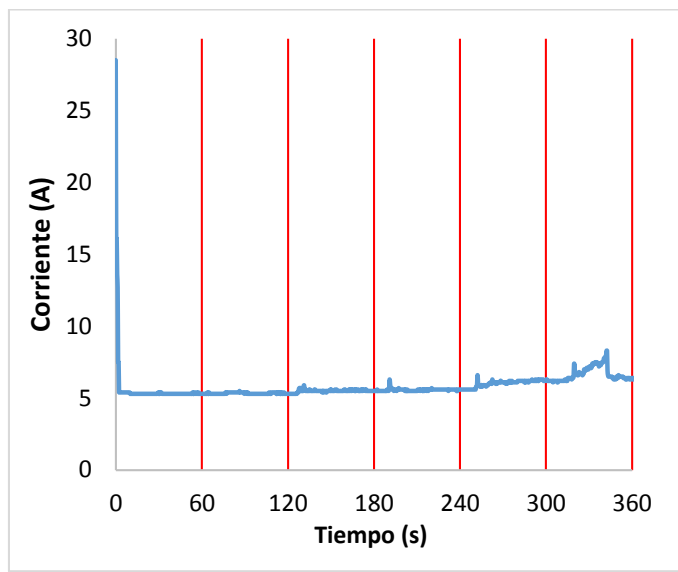

(D)

Figura 7. Resultados de los valores medidos en el quinto experimento con respecto al tiempo.

Modelo Matemático. En cada experimento, se seleccionó los picos más altos, luego se hizo la gráfica que se puede observar en la Figura 8, en la cual se observan los picos más altos de potencia con respecto a la carga de bagazo de caña que se arrojó en cada caso. Con esa gráfica se obtuvo la ecuación Ec 2 que describe la tendencia de la misma, la cual tiene una exactitud descrita en la ecuación Ec 3, que como se puede ver es demasiado precisa, casi exacta, debido a que el $\mathrm{R}^{2}$ es muy cercano a uno, razón por la cual se consideró como modelo de consumo de potencia del molino de martillo cuando tritura bagazo de caña de azúcar. 


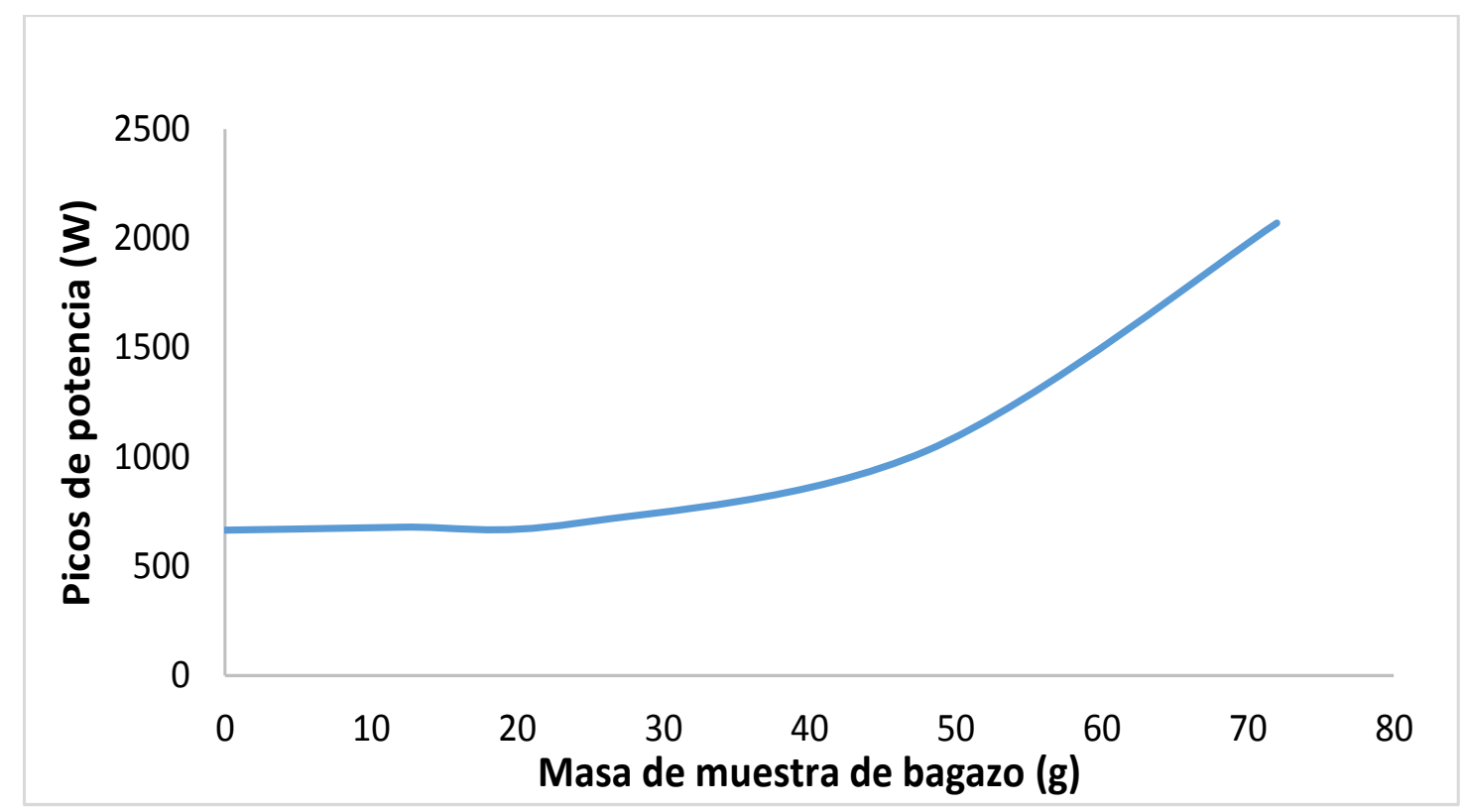

Figura 8. Picos más altos de potencia vs. el peso de cada carga de bagazo de caña de azúcar.

$$
\begin{aligned}
& P=0,4326 m^{2}-12,673 m+11,68 \\
& R^{2}=0,9911
\end{aligned}
$$

Donde:

$P$ : Potencia eléctrica utilizada para la molienda de las muestras,

$m$ : Peso de las muestras de bagazo de caña de azúcar.
Finalmente, con los polvos finos pesados en cada experimento se llegó a la Tabla 5, teniendo en cuenta que la bolsa donde se almacenaron pesa 17g. y la eficiencia de corte se define como la relación porcentual entre el peso de polvos sin bolsa y la masa total por prueba. Esta eficiencia no supera el $25,28 \%$ en el mejor de los casos, esto implica la acumulación del material faltante al interior del molino.

Tabla 5. Peso de los polvos finos después de la molienda.

\begin{tabular}{ccccc}
\hline $\begin{array}{c}\text { Masas por } \\
\text { muestra } \\
\text { (g) }\end{array}$ & $\begin{array}{c}\text { Masas totales } \\
\text { por prueba } \\
\text { (g) }\end{array}$ & $\begin{array}{c}\text { Peso de polvos } \\
\text { finos más bolsa } \\
\text { (g) }\end{array}$ & $\begin{array}{c}\text { Peso polvos finos } \\
\text { sin bolsa } \\
\text { (g) }\end{array}$ & $\begin{array}{c}\text { \% de eficiencia de } \\
\text { corte }\end{array}$ \\
\hline 12 & 60 & 27 & 10 & 16,67 \\
24 & 120 & 36 & 19 & 15,83 \\
48 & 240 & 69 & 52 & 21,67 \\
72 & 360 & 108 & 91 & 25,28 \\
\hline
\end{tabular}


Tener cuenta que la eficiencia de corte medida se hizo con la siguiente Ec 4:

$$
E f=\frac{M p f}{M t} x 100 \%
$$

Donde:

Ef : Eficiencia de corte

Mpf : Peso de los polvos finos que pasaron por la criba durante la molienda

Mt : Peso total de todas las muestras por experimento.

Hay que tener en cuenta que el material que no pasó por la criba se queda dentro de la máquina, debido a condiciones geométricas y fuerza de impacto del martillo, es decir, llega un momento en el cual una parte del bagazo obtiene una forma que el martillo no le es posible seguir triturando hasta el punto de que cruce la criba.

Para una mejor aclaración, la Figura 9 mostrará una comparación entre el total de cargas en cada experimento contra el peso de los polvos finos.

La medición de la potencia consumida por el molino de martillos en el tiempo, permite observar las variaciones de ésta, a medida que la carga de bagazo cambia en el molino. A partir de las mediciones de material procesado se observa que el material permanece hasta un $75 \%$ acumulado en el área de los martillos, ya que para el tiempo de experimentación solo se había procesado un $25 \%$. Esto hace que se incremente el consumo de potencia. Cuando se observó su potencia en condiciones de vacío se notan condiciones estables en el consumo de potencia posterior a la etapa de estabilización. Esta estabilidad se ve afectada a medida que son introducidas al molino cargas controladas de bagazo, reflejándose estas perturbaciones en la potencia consumida en dicho instante. Estas relaciones permiten evidenciar que el consumo de potencia se puede caracterizar a partir de su relación con la carga. El modelo presentado sugiere que esta relación es de tipo cuadrático con un coeficiente de correlación de 0,99.

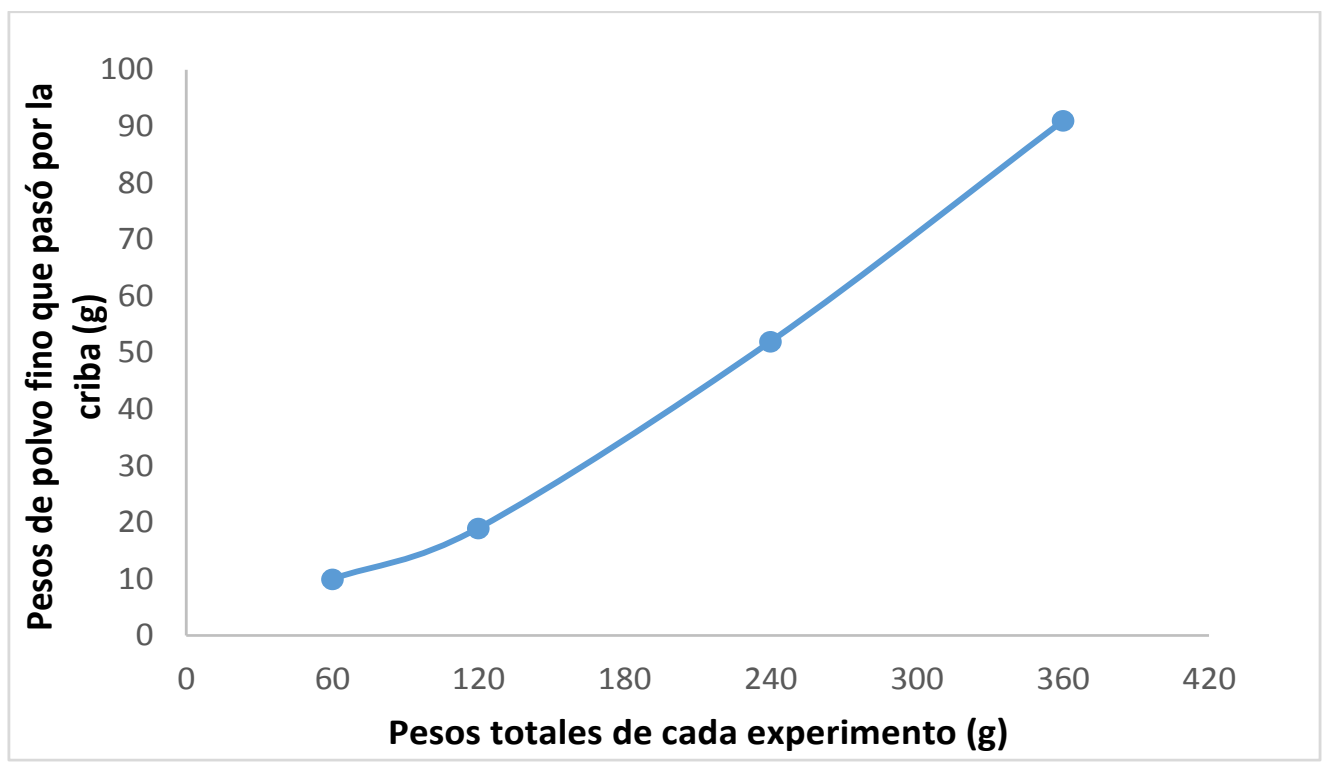

Figura 9. Relación entre los polvos finos que pasaron la criba durante la molienda contra el peso total de las muestras que fueron trituradas en cada experimento. 
En el estado del arte se identificaron dos artículos que presentan modelos matemáticos para el cálculo de la potencia en trituradoras, cuyo funcionamiento se asemeja al del molino de martillos. En el primero se presenta un modelo para el corte de pasto empleando cuchillas fijas al eje. Su modelo se basa en el cálculo de la velocidad de rotación y el ajuste de parámetros que contemplan las pérdidas por resistencia del aire, lanzamiento del material triturado, fricción en los apoyos y corte (Pérez y Garbati et al, 2008); aunque su modo de operar sea similar al del molino de martillos, ya que en ambos casos las cuchillas giran con respecto a un eje, más no es posible establecer una comparación, el motivo se debe a que las cuchillas en este modelo es fija y no contempla la fuerza por impacto por las cuchillas, algo que sí ocurre con del molino de martillos, debido a que en este caso, hay una holgura en los agujeros donde pasa el eje, esto hace que ocurra un movimiento pendular en los martillos durante el impacto; En cambio, en el otro caso, debido a que el eje y las cuchillas están completamente unidas, su modelo se puede tratar como una extrapolación al caso del fenómeno de corte en procesos de manufactura, (Martínez et al., 2004); este modelo contempla parámetros constructivos, cinemáticos que intervienen en este tipo de modelo y las propiedades físico-mecánicas del material procesado. Basado en las diferencias en ambos sistemas de corte, no es posible caracterizar la relación de corte del bagazo de caña con el molino de martillos usando el modelo de una trituradora de ejes fijos.

\section{CONCLUSIONES}

La eficiencia de corte del molino es directamente proporcional al caudal másico con el que arroja el material a triturar.

Entre mayor sea el caudal másico, el voltaje tiende a bajar, por ende, el coseno del ángulo de desfase tiende a aumentar, y la corriente aumenta.
Cuando el caudal másico es pequeño, la corriente y potencia tratan de estabilizarse durante el periodo de tiempo.

Este modelo muestra que existe una relación cuadrática entre las cargas y los picos de consumo de potencia.

Conflicto de intereses: Los autores declaran que no hay conflicto de interés.

\section{REFERENCIAS BIBLIOGRÁFICAS}

Agüero, A. C., Pisa, J. R., Agüero, C. J., \& Bugeau, A. T. (2004). Extensión Poder Calorífico del Bagazo de Caña de Azúcar. Revista CET, 13, 32-37.

Arpi Trujillo, J. E., \& Calderón Todal, C. S. (2010). Capítulo 3 análisis de las alternativas de los distintos sistemas peletizadores para la elección del más óptimo. In Diseño de una máquina peletizadora en base a la disponibilidad de residuos madederos de la ciudad de Cuenca para su aprovechamiento energético (pp. 80123).

Ayala Garay, A. V., Cervantes Osornio, R., Vargas Sállago, J. M., Benítez, A. A., \& Ramón Jiménez, R. (2013). Pruebas en tractores agrícolas en México para determinar la eficiencia a la toma de fuerza. (A. Y. Instituto Nacional de Investigaciones Forestales \& Pecuarias, Eds.). San Miguel Coatlinchán, México: Secretaría de Agricultura, Ganadería, Desarrollo Rural, Pesca y Alimentación.

Ballester, F. (2005). Contaminación Atmosférica, Cambio Climático y Salud. Rev Esp Salud Pública, 79, 159-175.

Bitra, V. S. P., Womac, A. R., Chevanan, N., Miu, P. I., Igathinathane, C., Sokhansanj, S., \& Smith, D. R. (2009). Direct mechanical energy measures of hammer mill comminution of switchgrass, wheat straw, and corn stover and analysis of their particle size distributions. Powder Technology, 193(1), 32-45. https://doi. org/10.1016/j.powtec.2009.02.010 
Bradshaw, J. (2009). Hammermills versus roller mills. Retrieved August 17, 2012, from https:// www.world-grain.com/articles/10011hammermills-versus-roller-mills

Byé, P., \& Fonte, M. (1992). Hacia técnicas agrícolas e base científica. Ensaios Fee, 13(2), 449-467.

Cañizares Arevalo, J. D. J. (2015). Tecnificación de la agroindustria panelera: Alternativa de empleabilidad de ingresos en convención, $\mathrm{N}$. de S. Modernization of Agroindustria Panela: Alternative and Income Employability in Convention, N. S. Revista Ingenio UFPSO, 08, 141-152. Recuperado de: http://revistas. ufpso.edu.co/index.php/ringenio/article/ viewFile/215/152

Chaparro Castro, J. M. (1995). Potencia en Máquinas Agrícolas. Ingeniería e Investigación, (32), 4-19. Recuperado de: https://revistas. unal.edu.co/index.php/ingeinv/article/ view/24803/25352

Chen, Z. ., \& Spooner, E. (2001). Grid power quality with variable speed wind turbines. IEEE Transactions on Energy Conversion, 16(2), 148154. https://doi.org/10.1109/60.921466

Durán, J. R., Insuasty, 0., \& Viveros, C. A. (2014). Comportamiento agroindustrial de diez variedades de caña de azúcar para producción de panela en Santander. Corpoica Ciencia y Tecnología Agropecuaria, 15, 183-195. https:// doi.org/https://doi.org/10.21930/rcta.vol15_ num2_art:358

Elias, E., Majaja, B. A., Ibrahim, S., \& Emmanuel, G. R. K. (2014). Noise Pollution In Maize Milling Smes. Tanzania Journal of Engineering and Technology, 35(1), 34-45.

Flórez, D. H. (2013). Agenda prospectiva de investigación de la cadena productiva de la panela y su agroindustria. Tecnura, 17(36), 72-86. https://doi.org/https://doi. orhttps://doi.org/10.14483/udistrital.jour. tecnura.2013.1.a04

Fredes Nuñez, N. A. (2014). Evaluacion Técnica y Económica de una Planta de Producción de Combustible Sólido a partir de Biomasa Forestal en la Región de Los Lagos. Chile: Universidad de Chile.
García, M. C. (2004). Hornillas paneleras evaluación de su impacto ambiental. Revista Corpoica. 4: 20-32.

Gracia, T. D., \& Mateo, G. F. (2016). Aprovechamiento del bagazo industrial de café como biomasa para la sustitución parcial de combustible. Ecuador: Universidad de Guayaquil.

Guerrero, M. E. \& Escobar, J. D. (2015). Eficiencia técnica de la producción de panela The technical efficiency of Non Centrifugal Sugar production. Revista de Tecnología - Journal of Technology, 14(1): 107-116.

Jannasch, R., Quan, Y. \& Samson, R. (2001). A Process and Energy Analysis of Pelletizing Switchgrass. Retrieved from https://reap-canada.com/ online_library/feedstock_biomass $/ 11 \% 20$ A\%20Process.pdf

Leiva, A. F., Tamara, L. V. \& Arbelaez, F. (2006). Sector panelero colombiano. Recuperado de http://www.panelamonitor.org/media/ docrepo/document/files/el-sector-panelerocolombiano.pdf

Martinez, P., Rodríguez I., Esperanza, G., \& Leiva, J. (2014). Caracterización y evaluación del bagazo de caña de azúcar como biosorbente de hidrocarburos. AFINIDAD, 71(53), 57-62.

Martínez A., Hernandez, Valdez, P., Díaz Suárez, J., Maturell, Y., \& Vega, D. (2004). Modelo matemático racional para el cálculo de la potencia consumida en molinos forrajeros de tambor. Revista Ciencias Técnicas Agropecuarias, 13(4).

Murcia-Pardo, M. L. \& Ramírez-Durán, J. (2017). Reconversión del sistema regional de producción de semilla de caña para la agroindustria panelera en Boyacá y Santander Regional. Corpoica Ciencia y Tecnología Agropecuaria, 18(1), 75-87. https://doi. org/10.21930/rcta.vol18_num1_art:559

Ozkan, B., Akcaoz, H. \& Fert, C. (2004). Energy input-output analysis in Turkish agriculture. Pergamon, 29, 39-51. https://doi. org/10.1016/S0960-1481(03)00135-6 
Pérez, M. S., \& Cortés, A. H. (2017). Efectos de la política de desarrollo rural en la economía campesina en Colombia 2002-2014. Bogotá: Universidad de La Salle.

Pérez, J. S., \& Garbati, F. (2008). Modelo matemático para la demanda de potencia de un aparato de corte de eje vertical para la trituración de rastrojos. Ingeniería E Investigación, 28(3), 4.

Rodríguez-Pérez, B., \& Contreras-Moya, RosaDomínguez, E. (2014). Comparación ambiental de la generación de energía eléctrica a partir del bagazo y fuel oil. Icidca, 48, 70-79.

Romantchik-Kriuchkova, E., Morelos-Moreno, Á., Villaseñor-Pereá, C. A. \& Pérez-Sobrevilla, L. (2015). Zonas de trabajo del conjunto del tractor agrícola con implemento en los esquemas de tracción 4x2 y 4x4. Agrociencia, 49(1), 53-67.

Santillán-Fernández, A., Santoyo-Cortés, V. H., GarcíaChávez, L. R., \& Covarrubias-Gutiérrez, I. (2014). Dinámica de la producción cañera en México: Período 2000 a 2011. Agroproductividad, 7(6), 23-29.

Tiwari, C. (2011). Producing fuel briquettes from sugarcane waste. In R. A. of Engineering (Ed.), Our Global Future. Londres, Inglaterra: Engineers Without Borders UK. Research \& Education.

Valdés, P. A., Martínez, A., Valencia, Y. \& Díaz, E. (2012). Validación del modelo de cálculo de la potencia consumida por las picadoras de forraje del tipo de tambor con alimentación manual. Tractores Y Máquinas Agrícolas, 21(2): 5-10. https://doi.org/10.4155/bfs.12.77

Vargas, J. M., Audelo, M. A., Jiménez, R., Ayala, A. V., \& Cervantes Osorno, R. (2013). Potencia y fuerza de tracción en la barra de tiro de los tractores agrícolas. Recuperado de: http://biblioteca.inifap.gob.mx:8080/jspui/ bitstream/handle/123456789/3950/ C I RCE_010208300100052501 ok. pdf?sequence $=1$

Vega-Nieva, D. J., Fernández, M., Ortiz, L. \& Corral-Rivas, J. J. (2015). Caracterización Bioenergética de los Residuos de Cosecha de las Principales Especies Forestales del Noroeste de España. Información Tecnológica, 26(4), 03-12. https://doi.org/10.4067/ S0718-07642015000400002

Vera A. (2014). Diseño de briquetas ecológicas para la generación de energia calórica y mejoramiento de ecosistemas en el corregimiento de Nabusimake, municipio de Pueblo Bello-Cesar. UNIVERSIDAD NACIONAL ABIERTA Y A DISTANCIA -UNAD. Recuperado de: https://repository.unad.edu.co/handle/10596/6111

Yancey, N., Wright, C. T. \& Westover, T. L. (2013). Optimizing hammer mill performance through screen selection and hammer design. Biofuels, 4(1), 85-94. https://doi. org $/ 10.4155 /$ bfs.12.77 